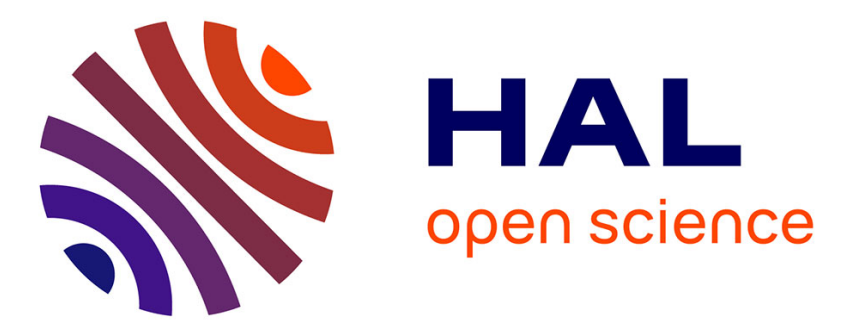

\title{
Les enfances de Perceval dans le Tristan en prose: jeunesse du héros et genèse du texte
}

Christine Ferlampin-Acher, Damien de Carné

\section{To cite this version:}

Christine Ferlampin-Acher, Damien de Carné. Les enfances de Perceval dans le Tristan en prose: jeunesse du héros et genèse du texte. Journal of the International Arthurian Society, 2013, 1, p. 50-80. hal-01846481

\author{
HAL Id: hal-01846481 \\ https://hal.science/hal-01846481
}

Submitted on 26 Jul 2018

HAL is a multi-disciplinary open access archive for the deposit and dissemination of scientific research documents, whether they are published or not. The documents may come from teaching and research institutions in France or abroad, or from public or private research centers.
L'archive ouverte pluridisciplinaire HAL, est destinée au dépôt et à la diffusion de documents scientifiques de niveau recherche, publiés ou non, émanant des établissements d'enseignement et de recherche français ou étrangers, des laboratoires publics ou privés. 


\section{Les enfances de Perceval dans le Tristan en prose : jeunesse du héros et genèse du texte}

Christine Ferlampin-Acher et Damien de Carné

L'édition du Tristan en prose a été l'une des grandes entreprises éditoriales qui ont renouvelé les études arthuriennes ces dernières années. Prenant la suite du travail mené par Renée Curtis sur le début du texte, ${ }^{1}$ les deux éditions collectives dirigées par Philippe Ménard ont permis l'accès aux versions majeures de ce roman protéiforme. Pragmatique et parfaitement averti de l'extraordinaire complexité de la tradition manuscrite du Tristan, l'éditeur écrivait dès les premières pages :

Il faudrait plusieurs vies humaines pour embrasser dans le détail tous les changements et les remaniements de l'œuvre. Comme d'autres textes du Moyen Âge, le Tristan en prose est en perpétuel devenir. Compte tenu de l'ampleur de la matière [...], il est radicalement impossible de se livrer à un examen minutieux des diverses rédactions et à des comparaisons méticuleuses entre les manuscrits. Nous ne parviendrions jamais au terme de notre entreprise.

${ }^{1}$ Le roman de Tristan en prose, éd. R. Curtis, 3 vols (I: München: Max Hüber, 1963 ; II: Leiden: Brill, 1976 ; III: Cambridge, Brewer, 1985). Fondée sur un manuscrit à certains égards remarquable, cette édition critique était sans doute impossible à achever : le manuscrit s'interrompt au quart du roman, ce qui n'empêchait pas l'éditrice d'affirmer qu'il n'était 'pas concevable d'envisager l'utilisation d'un autre manuscrit,' sans indiquer le parti à prendre pour la suite du texte ('Pour une édition définitive du "Tristan en prose"', in Cahiers de civilisation médiévale, 24 (1981), pp. $91-99$ (p. 99). Par ailleurs, le rythme de parution de l'édition, qu'elle assumait seule, repoussait à plusieurs décennies la publication du roman entier. 
[...] On est contraint de réduire ses ambitions et de limiter ses prétentions. L'important est de fournir un texte de bonne qualité qui servira de base aux recherches futures. ${ }^{2}$

De fait, les questions posées par ce roman n'ont pas toutes été résolues, loin s'en faut. Parmi les problèmes persistants, l'un des plus irritants est sans doute celui de l'appartenance proprement tristanienne de certains épisodes du texte. Nous aimerions revenir dans cet article sur le récit consacré aux enfances de Perceval tel que le donne la partie du Tristan éditée par Noëlle Laborderie et Thierry Delcourt (Champion II).

Ces aventures de Perceval sont présentes dans la partie du roman, qui, contrairement au début, diffère nettement dans les deux versions éditées. Si jusqu'à la quête de Tristan qui sui le tournoi du Château des Pucelles, le canevas est globalement le même dans tous les témoins -- abstraction faite des rajouts et raccourcissements --, ensuite deux traditions divergentes se dessinent clairement à partir de la libération de Tristan de la prison de Daras. ${ }^{3}$

${ }^{2}$ Le Roman de Tristan en prose, t. I, éd. Philippe Ménard (Genève: Droz, 1987), pp. 13-15. Ce volume et les huit autres qui le suivent (Droz, 1990-1997) donnent, là où l'édition Curtis s'interrompt, la 'version longue' du roman, traditionnellement désignée comme la 'deuxième version' (V.II) du texte, éditée à partir du manuscrit 2542 de la Bibliothèque Nationale de Vienne. La 'version courte' (V.I), qui se sépare de la version longue au milieu du t. III de l'édition Droz, a été publiée à la suite : Le Roman de Tristan en prose (version du manuscrit fr. 757 de la Bibliothèque Nationale de Paris), dir. Philippe Ménard, 5 vols (Paris: Champion, 1997-2007). Par commodité, nous renverrons aux différents tomes de ces éditions collectives sous la forme Droz I à IX, et Champion I à V.

${ }^{3}$ Eilert Löseth a fourni une analyse monumentale du Tristan : Le Roman en prose de Tristan, le Roman de Palamède et la Compilation de Rusticien de Pise (Paris: Bouillon, 1891). Il y a formulé l'idée d'une 'première' et d'une 'seconde' version, ou V.I et V.II (comme les a 
Les enfances de Perceval font partie de ces aventures qui sont absentes de la version longue, version qui cependant intègre largement la quête du Saint Graal, dont Perceval est l'un des héros. Elles sont au contraire racontées, avec originalité par rapport au Conte $d u$ Graal de Chrétien de Troyes et au Perceval en prose de Robert de Boron, dans la version courte, qui a tendance à évacuer le Graal mais ne peut s'en démarquer complètement, tant le poids de la tradition graalienne est forte.

La situation paradoxale du passage est renforcée par son appartenance tristanienne problématique : en effet cette série d'aventures, qui ne met à aucun moment Tristan et Yseut en scène, se retrouve à l'identique au sein d'un ensemble textuel plus complet (la Folie Lancelot) qui semble être une partie du Roman du Graal, ce cycle 'post-Vulgate' dont

désignées ultérieurement Emmanuèle Baumgartner, cf ci-dessous n. 5), qui divergent à partir du $§ 183$ de son analyse. Mais leur relation chronologique exacte n'est pas aussi certaine qu'il l'entendait. Les dénominations comme versions respectivement 'non-cyclique' et 'cyclique' ne sont pas très heureuses, étant donné que de nombreux traits cycliques sont communs aux deux versions. Appeler V.I 'version courte’ et V.II 'version longue’ est moins inexact, si cependant l'on garde à l'esprit que parfois V.II abrège ce que V.I développe, et que V.I présente des épisodes (d'origine ou rajoutés) omis par V.II. Quant au bien-fondé de la distinction des deux versions principales (Emmanuèle Baumgartner avait également proposé l'existence de versions plus tardives, V.III et V.IV, outre les versions particulières), elle a été ponctuellement remise en cause parce que certains épisodes censés les discriminer varient davantage en fonction des manuscrits qu'en fonction des 'versions' (ainsi des joutes de Tristan devant Arthur à son retour en Logres, voir éd. Champion, t. III, pp. 10-15 et variantes pp. 411-413). Cependant, elle demeure largement opératoire pour l'ensemble du texte, et en l'occurrence pour les épisodes que nous examinerons ici. 
Fanni Bogdanow a étayé l'existence. ${ }^{4}$ Dans son ouvrage fondamental sur la tradition et sur la signification du Tristan, Emmanuèle Baumgartner récusait l'origine tristanienne du passage (soutenue par Fanni Bogdanow), d'une part pour des raisons esthétiques (ces épisodes sont hors sujet dans le Tristan), d'autre part pour des raisons d'ordre philologique (mise en évidence d'allusions, de suppressions, de recompositions). ${ }^{5}$

Quatre décennies plus tard, la connaissance du texte et des manuscrits s'est accrue, mais la question est demeurée ouverte, et l'aporie irrésolue. Nous examinerons donc à nouveau les problèmes esthétiques et ecdotiques posés par les enfances de Perceval. Nous montrerons dans un premier temps qu'il n'y a pas de raison esthétique pour exclure cette série d'épisodes du scénario tristanien. Puis nous proposerons, avec la prudence qui s'impose faute d'éléments matériels nouveaux, de considérer cette série d'épisodes comme une part d'origine du Tristan en prose, mais dont les formes subsistantes sont déjà le fruit de réécritures préalables qui ont croisé le Tristan avec la 'post-Vulgate', les parties communes à ces deux textes remontant probablement à un original tristanien déjà refait. Mais une telle conception

${ }^{4}$ La Folie Lancelot. A Hitherto Unidentified Portion of the 'Suite du Merlin' Contained in Mss. B.N. fr. 112 and 12599, éd. Fanni Bogdanow, Beihefte zur Zeitschrift für romanische Philologie, 109 (Tübingen: Niemeyer, 1965). Sur le cycle supposé, voir son ouvrage The Romance of the Grail. A Study of the Structure and Genesis of a Thirteenth-Century Arthurian Prose Romance (Manchester University Press/New York: Barnes \& Noble, 1966), et surtout ses propositions plus récentes développées dans son édition de la dernière partie de la Post-Vulgate, La version post-vulgate de la Queste del Saint Graal et de la Mort le roi Artu, troisième partie du Roman du Graal (Paris: SATF, 1991-2001), t. I, pp. 29-48

${ }^{5}$ Emmanuèle Baumgartner, Le 'Tristan' en prose. Essai d'interprétation d'un roman médiéval, Publications Romanes et Françaises, 133 (Genève: Droz, 1975), pp. 41-48. 
oblige à repenser plus largement le mode de composition et l'identité même du Roman de Tristan en prose, et avec lui de la 'post-Vulgate'.

\section{La place des enfances de Perceval dans V.I et V.II}

L'apparition de Perceval dans le Tristan se produit très différemment dans les deux éditions, bien qu'elle soit dans les deux cas inspirée du récit qu'en fait le Lancelot en prose. Dans le texte du manuscrit de Vienne, support de l'édition de V.II, la jeunesse de Perceval est évoquée à deux reprises, d'abord dans le t. IV de l'édition (à partir du § 140), puis dans le t. VI (à partir du § 55). Cette deuxième apparition ne nous intéressera pas : les chapitres de préparation à la Quête dans lesquels elle se situe, empruntés mot pour mot au Lancelot, sont un ensemble adventice ajouté ultérieurement au texte de V.II. Selon Emmanuèle Baumgartner, leur présence est dans les autres manuscrits un indice d'appartenance à V.III, que le manuscrit de Vienne rejoint donc là temporairement (Droz, t. VI, p. 32-36). Le récit qui concerne Perceval a été repris sans précaution au texte-source et contredit à la fois la chronologie des parties précédentes du roman et le contenu diégétique de la première occurrence de l'arrivée de Perceval.

Celle-ci, dans le t. IV (pp. 32-36), est annoncée par une double formule d'entrelacement ( $§ 138$ et 139). D'abord, après l'évocation de la haine de Gauvain pour Lamorat, on assiste au départ de Camelot de Tristan et Marc. Arthur et sa cour, inquiets pour le héros, sont plongés dans le chagrin, pendant que Marc nourrit de sombres pensées. Une longue formule d'entrelacement annonce alors les débuts de Perceval, nouveau venu dans le récit : 
Mais atant laisse ore li contes a parler du roi March et de monsigneur Tristan, car bien i savra retourner quant il en sera lieus et tans, et retourne au roi Artu pour conter des oevres des chevaliers ki estoient repairans en son ostel et pour deviser conment ne en quel maniere Percevaus vint a court premerainnement ne qui l'i amena. Pour ce que je ne voel pas laissier que je ne vous conte ceste aventure et pour ce d'autre part que a nostre matere apartient de conter, si vous dirai conment il vint laiens premierement et conment li rois Artus le fist chevalier, et dirai en tel maniere (Droz IV, § 138, 11. 15-26).

Mais le récit demeure brièvement attaché à la cour. Perceval n'apparaît pas dans ce très court chapitre (une seule page) qui se conclut par l'annonce de l'emprisonnement de Tristan et par une nouvelle formule d'entrelacement, la seconde à annoncer Perceval :

Mais atant laisse ore li contes a parler de celui fait, car bien i savra retourner quant tans en sera, et retourne a nostre matere pour deviser conment Percevaus vint a court, pour ce que nous le vous avom en couvenent de deviser (ibid., § 139, 11. 33-37).

Le récit enchaîne alors sur l'arrivée de Perceval et Agloval son frère à la cour et sur l'adoubement du premier, qui disparaît cependant du récit pour laisser la place à la mention des succès de son frère Lamorat. Le fait que Perceval soit amené à la cour par son frère Agloval et qu'Arthur le fasse chevalier le lendemain s'appuie bien sur les données du Lancelot en prose ${ }^{6}$ mais la reprise n'est pas littérale. Le rédacteur s'inspire de loin de son modèle pour insister surtout sur la valeur du lignage de Perceval, soulignée par Keu. D'emblée l'identité, le lignage et la noblesse des frères sont ostensiblement marqués (gentius est répété quatre fois en quelques lignes) : Et sachiés que chil Perchevaus fu fieus le roi

\footnotetext{
${ }^{6}$ Lancelot, roman en prose du XIII ${ }^{e}$ siècle, éd. Alexandre Micha, 9 vols (Genève: Droz, 19781983), t. VI (1980), § 23.
} 


\section{Pellynor de Lystenois et frere de Lamorat de Gales et de ses autres freres, et estoit li}

mainsnés (ibid., § 140, 11. 30-33). Une fois chevalier, Perceval sort du récit jusqu'à la Quête.

On le retrouve dans l'allusion de la fin du volume à la libération de Tristan (ibid., § 248), puis

dans le tome suivant, mentionné rapidement dans des aventures chevaleresques (contre

Bréhus, Droz V, § 17) ou lorsque Keu constate son absence à la cour (ibid., § 184).

La double formule d'entrelacement, l'étrangeté de cet appendice d'une seule page qui oblige le narrateur à répéter son annonce de l'arrivée de Perceval, enfin la gratuité de cette éphémère apparition de Perceval suggèrent que V.II a remanié le texte original. Le redoublement formulaire à si peu de distance apparaît comme un dysfonctionnement du procédé de l'entrelacement, qui traduit sans doute la gêne du prosateur devant cette matière qu'il lui était difficile de ne pas mentionner mais dont il ne souhaitait pas s'occuper. ${ }^{7}$

Il n'y a donc pas d'histoire de Perceval racontée dans V.II, simplement une mention qui cache mal le recours à une source plus étendue. Le récit de V.I, en revanche, développe les aventures du personnage.

Il intègre sa rencontre avec Agloval (qui réécrit en partie le Conte du Graal et le Lancelot) dans une longue séquence centrée sur la quête de Lancelot fou. Juste avant l'apparition de Perceval, ce sont les aventures de Driant et Lamorat, ses frères, et leur

\footnotetext{
${ }^{7}$ Cf. D. de Carné, Sur l'organisation du Tristan en prose (Paris: Champion, 2010), pp. 113114 et 151 : 'La disjonction entre l'annonce contenue dans le seuil et la matière du segment suivant correspond exactement à une part de V.I que V.II tient à distance [...]. Les seuils désignent ainsi, par une référence allusive, la matière à laquelle le prosateur de V.II a refusé l'accès à son roman'.
} 
assassinat par Gauvain. ${ }^{8}$ Dans V.I, on trouve aussi un double système d'annonce concernant Perceval. Au début de la quête de Lancelot est en effet annoncée sa délivrance par Perceval :

por ce que $[. .$.$] il delivra monseigneur Lancelot [\ldots]$ nos convient il trere vers celui conte qui devise la vie de lui. Et nos deviserons ore premierement coment il fu amenéz a cort [...]. Vérités fu, et l'estoire veraie le devise, que li rois Pellinors, qui fu pere Perceval, ot .V. fiz. (Champion II, § 3, 20-§ 4, 1)

Suivent l'origine et l'histoire de la haine entre Gauvain et Lamorat, sur 70 pages, avant qu'une nouvelle formule d'entrelacement n'introduise l'histoire de Perceval que l'on nous avait promise :

Si en lesse orez atant li contes a parler et retorne a Agloval por deviser conment il mena Perceval, son frere, a la cort le roi Artus.

Or dit li contes qe bien tint Agloval... (ibid., $§ 49,81-\S 50,1$ )

Pourtant, à la différence de V.II, où la double formule d'introduction ne semble pas correspondre à un projet narratif précis mais à l'incapacité de quitter en une fois le sujet brûlant du départ de Tristan, la double annonce se justifie ici par la volonté de creuser le

${ }^{8}$ V.II ne raconte pas ces aventures, mais résume (Droz IV, § 248) : 'En chelui tans ochist sans doute mesire Gavains Lamorat de Gales, le boin chevalier. Ce fu damages et dolours grant pour toute la cevalerie du monde. Et sacent tout que, un poi de tans avant que Lamorat fust ochis, prist li rois March monsigneur Tristan en traïson et le fist metre en prison. Et le tint illuex si grant tans a grant dolour et a grant mesaaise que Percevaus de Gales vint en Cornuaille et delivra monseigneur Tristan...' 
passé, de montrer avec quelle lourde hérédité Perceval arrive à la cour, chargé d'une histoire familiale et d'une haine lignagère.

V.I ne présente donc pas l'agrammaticalité narrative de V.II dans cet épisode. Les apparitions de Perceval laissent penser que V.II s'est dissociée soit de V.I, soit d'une source que V.I suivrait de plus près. Le prosateur de V.II laisse même voir la tentation qu'il a probablement ressentie de purement et simplement supprimer Perceval de toute cette partie : Pour ce que je ne voel pas laissier que je ne vous conte ceste aventure et pour ce d'autre part que a nostre matere apartient de conter... La double justification se formule en termes éloquents : ce qui nous reste de Perceval dans V.II est ce que le prosateur a bien voulu ne pas laissier de côté. La protestation selon laquelle Perceval appartient bien à la matere du récit, protestation redoublée par le seuil suivant, ressemble à une tentative assez fruste de persuader le lecteur que Perceval a bien sa place ici -- voire de s'excuser de l'y avoir laissé, tenu par sa source.

Il n'y a donc pas de raison de considérer que Perceval n'était pas censé, dans le Tristan, délivrer le héros éponyme de son emprisonnement (témoin le résumé de cet épisode donné par V.II : Droz IV, § 248, cf. ci-dessus n. 8), donc pas de raison a priori de douter de l'appartenance proprement tristanienne de l'histoire de Perceval.

Le décentrement que subit V.I, du fait de l'introduction de ces dizaines de pages sans son héros, n'est pas une raison suffisante pour exclure celles-ci du texte tristanien 'originel'.9

\footnotetext{
${ }^{9}$ Emmanuèle Baumgartner, réfutant le point de vue de Fanni Bogdanow : 'Je comprends mal les raisons de ce subit intérêt de l'auteur du Tristan pour l'histoire de Perceval, personnage qui, exception faite de cet épisode très marginal de la folie de Lancelot et du rôle assez inattendu qu'il joue dans la délivrance de Tristan emprisonné par Marc, ne réapparaît
} 
À ce stade du récit, en effet, Tristan est en prison. Or le Tristan en prose a déjà recouru au même procédé : le protagoniste étant immobilisé pendant une longue période, le temps du roman se remplit d'aventures qui mettent en scène d'autres acteurs, sans lien nécessaire avec la destinée de Tristan. C'était le cas lors de la folie de Tristan, où le récit suivait les aventures de Kahédin jusqu'à sa mort, ainsi que, selon l'ordre des épisodes adoptés dans les différents manuscrits, celles du Chevalier à la Cotte Mal Taillée. ${ }^{10}$ C'était également le cas lors de l'emprisonnement de Tristan chez Daras, dans le t. III de l'édition de V.II, qui laissait le champ libre à Keu, Yvain aux Blanches Mains et Gaheriet en Logres et en Cornouailles, pendant près d'une centaine de pages (Droz III, $\S \S 46-149$; cette section appartient encore au Tristan commun).

Sur ce point, les seuils formulaires de V.II cités plus haut donnent des indices précieux. Rappelons-nous que le premier disait vouloir retourne [r] au roi Artu pour conter des oevres des chevaliers ki estoient repairans en son ostel : cela ne décrit-il pas exactement une partie du récit consacrée non pas à Tristan mais aux péripéties relatives à la cour d'Arthur en général, que développe V.I ?

pratiquement pas dans le Tristan proprement dit ni même dans la version de la Queste que donnent les manuscrits de V.I.' (Essai d'interprétation, p. 42)

${ }^{10}$ Voir éd. Curtis t. III, pp. XXI-XXIV ; Droz I, p. 21 ; ainsi que Renée Curtis, 'A romance within the romance: the place of the "Roman du Vallet a la Cote Maltailliee" in the Prose Tristan', in Mélanges Brian Woledge, (Genève: Droz, 1987), pp. 17-35. La question de savoir si les aventures de Brunor étaient d'une pièce ou entrelacées dans leur version d'origine n'est pas tranchée mais l'absence de Tristan pourrait être un élément de réflexion complémentaire par rapport aux hypothèses déjà avancées. 
Au-delà de la seule nécessité du remplissage narratif, l'essentiel est que les enfances de Perceval, telles que V.I nous les raconte, sont placées sous le signe de la vendetta entre les lignages de Lot et de Pellinor. Perceval survient dans une atmosphère de haine lignagère, après la mort de Lamorat et celle de Driant, comme pour combler le vide laissé par celles-ci. Les deux frères sont enterrés l'un à côté de l'autre (Champion II, § 49), ce qui rappelle pour l'éternité la rivalité des deux lignages. Perceval était mentionné comme celui qui a libéré Lancelot et Tristan (et c'est pourquoi nos convient il trere vers celui conte qui devise la vie de lui. Et nos deviserons ore premierement coment il fu amenez a cort, etc., ibid., § 3), mais le texte fait précéder l'apparition du tout jeune homme par des épisodes de la rivalité haineuse des lignages de Pellinor et de Lot, et raconte comment Gauvain a tué Lamorat (ibid., §§ 5-49), alors que quatre chevaliers du lignage de Pellinor (Agloval, Tor, Drianz, Lamorat) s'étaient mis en quête de Lancelot. Jaloux, offensés par les amours de leur mère avec Lamorat, Gauvain et ses frères (excepté Gaheriet qui cependant eut la malchance de trouver sa mère en compagnie de Lamorat et la tua, p. 67) haïssent le lignage de Pellinor. Perceval n'a toujours pas été présenté et ce n'est qu'après le récit de la mort de Lamorat que la seconde formule d'entrelacement qui annonce Perceval nous amène effectivement le personnage.

Les chapitres concernés sont hantés par l'inexorabilité de la mort de Lamorat, tué par Gauvain. Cette atmosphère de discorde entre les chevaliers, empesée par les promesses de mort et de vengeance, rappelle fortement La Mort le roi Artu. Et c'est un fait que, tant dans V.I que dans V.II, cette partie du récit, à partir de l'arrivée de Marc à la cour d'Arthur, adopte une tonalité descendante qui emprunte à la Mort Artu. D'ailleurs, le rôle de Tristan dans la délivrance des jeunes filles condamnées au bûcher par Arthur démarque sans discrétion excessive le sauvetage de Guenièvre par Lancelot dans le mouvement final de la symphonie arthurienne : la quasi citation explicite la parenté entre les deux textes et met l'accent sur les divisions qui envahissent la cour d'Arthur, et sur les limites des usages de la société 
arthurienne. ${ }^{11}$ Les péripéties ultérieures mises en scène par V.I, agressions fratricides entre chevaliers pourtant liés par la Table Ronde, s'inscrivent dans le prolongement de ces menaces nouvelles.

Par ailleurs, cette guerre des lignages a peut-être partie liée avec la déconsidération du modèle graalien, qui est une ambition manifeste de l'ensemble du Tristan en prose. ${ }^{12}$ Juste avant qu'il ne soit question de Perceval, le meurtre de Lamorat par Gauvain donne lieu à une scène originale : la tête de Lamorat, qu'a coupée Gauvain, est portée dans un bel vessel d'argent (§ 49) à la cour d'Arthur, à Quimpercorantin, par un ermite qui la présente au roi, sur le mode de l'antiphrase : 'Rois Artus, veez ci les bonnes œuvres qe ti parant font es questes aventureuses !' avant d'appeler la vengeance de Dieu. Au roi qui le presse de dénoncer le meurtrier, l'ermite refuse de répondre. Il quitte les lieux, tandis qu'Arthur devine que le coupable est Gauvain. Le 'vessel' funèbre est un double sombre, profane, tragique, du Graal :

${ }^{11} \mathrm{C}$ 'est le passage de Marc qui oblige Arthur à instaurer le serment judiciaire, la coutume existante s'étant avérée incapable de garantir la justice ('et fu dit a chelui point que desloiautés avoit mis loiauté au dessous ; par coi li serement des batailles fu adonc mis avant premierement', Droz IV,$\S 88$ ). Sur l'opposition idéologique entre le monde d'Arthur et ses ennemis, voir Emmanuèle Baumgartner, 'Arthur et les chevaliers envoisiés', in Romania, 105 (1984), p. 312-325.

${ }^{12}$ Sur les rapports entre le Tristan en prose et le Graal, l'ouvrage de référence demeure celui de C.-A. Van Coolput, Aventures querant et le sens du monde. Aspects de la réception productive dans les premiers romans du Graal cycliques dans le Tristan en prose (Louvain: Presses de l'Université, 1986). Les travaux ultérieurs consacrés à ce sujet, notamment les volumes de l'édition de V.I, ont établi que V.I et V.II adoptaient certes des moyens différents (V.II intègre et intercale le texte de la Queste pour mieux le neutraliser ; V.I l'exclut mais se livre à une relative déconsidération de Galaad) pour un but toutefois similaire. 
porté cérémonieusement, il contient un reste humain (comme le Graal a contenu le sang du Christ), qui est l'objet d'un mystère, d'un questionnement, et qui renferme le fin mot -- ici dévoyé -- des quêtes aventureuses. ${ }^{13}$ Mais au lieu d'une quête mystique, la problématique est celle de l'amour adultère et de la vendetta ; l'ermite n'est pas un guide spirituel qui diffuse la bonne parole, mais une simple utilité qui parle par antiphrase. Cette scène qui, en convoquant les modèles d'Holoferne et Jean-Baptiste, attire l'attention sur les passions individuelles néfastes et sur la responsabilité féminine, celle de la mère de Gauvain en l'occurrence, étouffe les promesses spirituelles du Graal. Les préoccupations ici véhiculées sont violemment contradictoires avec celles de la future Quête : ce regard dessillé sur la pertinence des idéaux chevaleresques, contrepoint quasiment ironique de l'ambition spirituelle de la Queste del Saint Graal, s'inscrit fort bien dans la perspective générale du Tristan.

On comprend dès lors que l'enfance de Perceval, que Chrétien de Troyes avait présenté comme un orphelin victime des excès de la vie chevaleresque, ait trouvé sa place. On va voir en outre que la réécriture opérée, aussi bien du Conte du Graal que du Lancelot, confirme les traits que nous venons de mettre en valeur. ${ }^{14}$

\section{La réécriture du personnage}

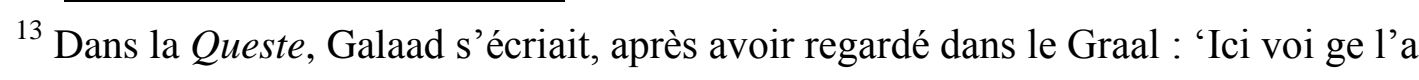
començaille [sic] des granz hardemenz et l'achoison des proeces; ici voi ge les merveilles de totes autres merveilles !' (éd. Albert Pauphilet (Paris: Champion, 1923), p. 278).

${ }^{14}$ Cette réécriture a déjà été étudiée par le passé : Alfons Hilka, 'Die Jugendgeschichte Percevals im Prosa Lancelot und im Prosa Tristan', in Zeitschrift für Romanische Philologie, 52 (1932), pp. 513-536, où l'auteur édite les fols 64'-68' du BNF fr. 757. 
Entre la célèbre scène de la rencontre des chevaliers par Perceval, dans le roman de Chrétien, et la rencontre de Perceval et Agloval dans V.I, certains détails témoignent d'une reprise précise : Perceval lance ses javelots 'une ore arriere et oltre avant, | une ore an bas et altre an haut' (98-99) dans le texte en vers, ${ }^{15}$ 'une heure avant et l'autre arrieres, une en haut, autre bas’ dans le texte en prose (§ 52). Il demande à son interlocuteur : 'Estes Dex ? - Nenil, par foi. |-Qu'estes vos dons ? Chevaliers sui' (172-173), ce qui est repris ainsi en prose : 'Ce vos n’estes dont Dieu, qe estes vos dont ? - Je sui, fet il, un chevalier' (§ 53). Outre ces échos, on note un effort, typique de la prose, de réorganisation des données : chez Chrétien, Perceval prend les chevaliers pour des anges de lumière (136), puis il demande à son interlocuteur s'il est Dieu (172), alors que dans la prose il prend Agloval pour 'ou Dieu ou angre', et la question qu'il pose correspond exactement à cette alternative (§ 52). La triple reprise, insistante, souligne l'effort de correction que le remanieur accomplit. De même l'éclat des armes au soleil est expliqué dans la prose par le fait que ces armes sont neuves (ibid.), ce qui est absent des vers de Chrétien et correspond à la visée 'rationalisante' que manifestent parfois les textes en prose -- tous ces éléments sont malgré tout absents du Lancelot.

La réécriture cependant modifie considérablement le sens de l'épisode. Alors que dans le roman en vers, le jeune garçon ignore son nom, dont la révélation sera l'enjeu d'une construction narrative complexe, ici il est identifié d'emblée -- un peu plus vite que dans le Lancelot -- par Agloval comme son frère : son histoire ne sera pas une quête identitaire. Prolongeant cet acquis du Lancelot, V.I retient des vers surtout l'idée d'un lignage qui a été décimé par la mort, idée qui implique une mise en cause de la chevalerie, responsable de la douleur de la mère. L'auteur ajoute d'ailleurs un long développement sur la douleur de celleci (§ 51, 11. 3-59), juste avant la rencontre entre Perceval et Agloval. Cet ajout, tragiquement, annonce la fin. Par ailleurs, comme la prose omet certains détails pittoresques donnés par

\footnotetext{
${ }^{15}$ Chrétien de Troyes, Le Conte du Graal, éd. F. Lecoy, 2 vols (Paris: Champion, 1973), t. I.
} 
Chrétien (la reverdie, le vacarme des armes...), tout est placé d'emblée sous la signe de la douleur de la mère, du lignage qui ne saurait rester mystérieux, du nom qui pèse comme une fatalité, d'une vendetta qui dessine un destin loin du Graal. La scène est centrée sur le lignage de Perceval et la mère douloureuse, dont le suicide possible est évoqué crûment (§ 59). Désormais, le père de Perceval est bien identifié : c'est Pellinor, et le lecteur, informé par ce qui précède dans le récit, sait que les deux frères, anonymes chez Chrétien, sont Driant et Lamorat, qui sont morts et qu'il faudra venger.

Le texte enchaîne alors avec une réécriture du Lancelot en prose : l'arrivée d'Agloval chez sa mère (adaptée puisqu'il a déjà rencontré Perceval), le séjour chez celle-ci, le départ de Perceval, la mort de la mère et le premier séjour du jeune garçon à la cour d'Arthur (cf. éd. Micha, t. VI, § 23). L'enchaînement est préparé en amont, dans l'épisode précédent, par la substitution d'Agloval, le frère de Perceval, aux cinq chevaliers anonymes de Chrétien de Troyes. Quand Perceval lui est présenté, le roi formule des remarques sur son glorieux lignage et sa parenté avec Pellinor, ignorée du Lancelot, ainsi que les larmes, le matin de l'adoubement, versées par ceux qui ont connu son père et Lamorat. Ces détails, tout comme la scène où Arthur demande à Gauvain et Gaheriet leur avis sur le beau jeune homme et prédit qu'il vengera Lamorat, Driant et son père, confirme le lien entre les débuts de Perceval et la vendetta familiale. Par ailleurs, toujours soucieux d'expliquer, de rationaliser, l'auteur du Tristan clarifie l'allusion, un peu confuse, du Lancelot aux trois tables de la cour ( $\$ 62$ : celle de la Table Ronde, celle des autres chevaliers et celle des chevaliers sans réputation), grâce à une intervention explicite de la voix conteuse qui montre le rédacteur en interaction avec sa source ('Et sachiés que...'). Malgré ces différences, l'épisode de la demoiselle muette est suivi d'assez près : le rédacteur semble avoir eu son modèle sous les yeux, tant certaines formulations sont proches : 
Et lors en avint une merveille grant qi fu tenue a grant miracle, et si dut il bien estre, car la damoisele qui oncquez n'avoit parlé, si conme cil de la meson le roi Artus le savoient, parla adont et dit : 'Perceval, serjant de Jesus Crist, virge chevalier et net, lesse celui siege ou tu te siez, et t'en vien seroit dejoste le siege Perilleux (Champion II, § 63)

Et lors en avint une aventure mervilleuse qui fu tenue a miracle et si dust ele estre, car celle qui onques n'ot parlé dist à Perceval : 'Perceval, serjanz Jhesus Crist, vierges et nez, vien toi seoir ou haut.' (Lancelot, loc. cit., § 24).

Le Tristan, comme son modèle, conduit Perceval sur le siège qui est à côté du Siège Périlleux, lui fait annoncer que ce siège est promis au meilleur chevalier à venir et que Bohort sera à sa gauche, puis laisse mourir la demoiselle, munie des saints sacrements, dans une chambre voisine. Cependant l'auteur ajoute une allusion explicite à la 'grant qeste du Saint Graal' (§ 63, 1. 67) qui n’est pas encore commencée.

Mais elle ne semble annoncée que pour être ensuite confinée, jusqu'à l'asphyxie, dans une problématique de vendetta qui, portée par un temps qui s'allonge, se nourrit de haines recuites. Retenu à la cour, Perceval ne la quitte, dans le Lancelot et dans V.I, que 1'hiver suivant: Le Tristan reprend alors deux éléments du Conte du Graal : la prédiction du fou et les gouttes de sang sur la neige (voir dans Chrétien les séquences commençant aux vers 1248 puis 4142, éd. cit.). Dans le Tristan comme dans le Lancelot, Keu et Mordret se moquent de Perceval dont l'écu ne porte pas de trace de coups, et un fou répète ces propos (§ 64) : vexé, Perceval décide de partir en aventure et ce sera, dans le Tristan, l'épisode des gouttes de sang. La prose laisse encore entendre quelques expressions présentes dans les 
vers. ${ }^{16}$ Cependant, dans V.I, Perceval, dérangé dans sa rêverie, se bat successivement contre Keu, Mordred et Gauvain, alors que Chrétien le confronte à Sagremor, Keu et Gauvain. Keu était imposé par l'épisode du fou. Mordred quant à lui est préféré à Sagremor, car c'est un frère de Gauvain. Ainsi le Tristan épaissit la rivalité entre les deux lignages et accumule les griefs entre eux : Gauvain, Mordred et Agravain, qui par ailleurs soupçonnent que Perceval veut venger Driant et Lamorat $(\S 72)$, sont plus que jamais animés de haine contre lui à la suite de ses victoires dans l'épisode des gouttes de sang : 'Ensint s'acordent li troi frere a la mort de Perceval et dient que il n'avront jamés ne bien ne repos devant que i l'aient ocis’ (§ 73).

Une autre différence est le nom de la dame des rêveries du héros : le sang sur la neige rappelle à Perceval, non Blanchefleur, que le texte ignore, mais Hélaine sans Pair, dont le texte n'a pas encore parlé :

Et maintenant li sovient d'une dame que il avoit veüe n'avoit pas lonc tens au roiaume de Norgales, et estoit celle dame appellee Halayne sanz Per, et se estoit sanz doubte toute la plus belle dame du monde, fors la roÿne Guenievre. (§ 66)

Le nom de la dame, Hélaine sans Pair, s'inscrit dans la même logique. Certes il n’a jamais été question d'elle auparavant et l'on ne voit pas où ni quand Perceval, au cours de sa

\footnotetext{
16 'La noif estoit blanche a merveilles et li sanc estoit vermaux encontre, si que la blanchor responnoit au vermeil et donnoit une si vive color et si naturel que ce estoit merveilles a veoir.' (§ 66), cf. Chrétien : 'la noif qui soz la gente jut | et la sanc qui ancor parut [...] | si seinna .III. gotes de sanc | qui espandirent sor le blanc, | si sanbla natural color [...] | li vermaux sor le blanc asis | come les gotes de sanc furent | qui desor le blanc aparurent.'
} (4165-4184). 
toute jeune carrière a pu la rencontrer. Pourtant la substitution est significative. Tout d'abord, Helaine sans Pair est présente dans le Lancelot en prose (éd. cit., t. VIII, § 55) : ainsi au cœur même de la récriture du Conte du Graal en vers cette insertion permet de resserrer le maillage entre les deux textes. Mais c'est par ailleurs dans le Perceval en prose de Robert de Boron que Perceval aime Elainne et en est aimé. La jeunesse de Perceval, dans le Lancelot ou le Tristan n'a guère à voir avec ce texte, qui annonce dès le début que Perceval est le fils d'Alain le Gros, auquel il succède comme roi du Graal. Cependant notre version lui emprunte les amours de Perceval avec Elaine, sans les raconter, sur le mode de l'allusion. Or cette Elaine est, dans le texte attribué à Robert de Boron, la sœur de Gauvain. ${ }^{17}$ Aux amours de Perceval et Elaine, une sœur de Gauvain, le Tristan pouvait réserver une mention : de même que Lamorat, du lignage de Pellinor, a aimé la mère de Gauvain, de même Perceval, son frère, aime sa sœur. L'amour d'Hélaine, même si Tristan ne le développe pas, même s’il n'est qu'une allusion, suffit pour enfler, encore, la rivalité entre les deux lignages. ${ }^{18}$ D'ailleurs le

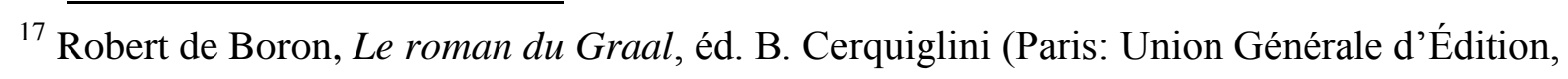
1981), p. 201 ; The Didot Perceval, éd. W. Roach (Philadelphie: University of Pennsylvania Press, 1941), p. 145. Pierre Gallais rapproche le nom d'Elaine-Hélène de celui d'Alain, le père de Perceval et propose une origine orientale ('Robert de Boron en Orient', in Mélanges Jean Frappier (Genève: Droz, 1970), t. I, p. 318).

${ }^{18}$ Ajoutons toutefois qu'Elaine n'est pas 'sans Per' dans le Perceval en prose, même si elle est la plus belle. C'est dans le Lancelot en prose qu'apparaît une Hélaine sans Pair : enfermée dans une tour par son mari Persidès, elle est défendue par Hector, qui la réconcilie avec son époux (voir éd. cit., t. VIII, p. 393). Si donc l'amie de Perceval nommée Elaine vient du Perceval en prose, le qualificatif vient du Lancelot. Or dans le Lancelot, Perceval, quittant la cour après sa première visite (éd. cit., t. VI, § 29-32), délivre un chevalier nommé Patridès (pendant que dans le Tristan il contemple les gouttes de sang sur la neige qui lui font penser à 
manuscrit BNF fr 103, qui donne une version isolée du Tristan en prose, racontera comment Perceval a pu admirer Hélayne sans Pair, la sœur de Gauvain, à la cour d'Arthur et se faire aimer d'elle -- les remanieurs ont souvent eu à cœur de combler les manques narratifs des versions précédentes. ${ }^{19}$

La réécriture des enfances de Perceval est ainsi complètement orientée vers le rappel des tensions entre lignages qui assombrissent le paysage arthurien en ce lieu du roman. Le Graal est escamoté : le texte réécrit Chrétien de Troyes, de la scène inaugurale de la rencontre avec les chevaliers jusqu'aux gouttes de sang, mais omet l'épisode du château du Graal, qui chez Chrétien, se déroule entre les deux. Le personnage de Perceval, comme l'a vu Fanni Bogdanow, ${ }^{20}$ est fondamentalement revu. De même que la préhistoire arthurienne du Tristan rattache Tristan au lignage sacré de Joseph d'Arimathie, mais par le personnage insoumis de

Hélaine). Patridès et Persidès ont pu être confondus ; ainsi, dans la liste des quêteurs du Graal donnée par V. II (Droz VI, § 112, 1. 66), les noms de Percidès et Patridès alternent (ibid., index des noms propres, p. 453). Si cette confusion est possible pour les lecteurs médiévaux, il peut sembler logique que Perceval, en quittant la cour, ait dans le Tristan une aventure en relation avec Hélaine, que le Lancelot présente comme la femme de Persidès, tandis qu'au moment analogue du Lancelot il délivre Patridès (Droz VI, p. 195)...

${ }^{19}$ E. Löseth, Le Roman de Tristan, § 282c. Sur la version du ms BNF fr. 103, voir Emmanuèle Baumgartner, Essai d'interprétation, pp. 77-83.

20 'The transformation of the role of Perceval in some thirthteenth century prose romances', in Studies in medieval literature and languages in memory of Frederick Whitehead, éd.

W. Rothwell, W. R. J. Barron, D. Blamires et L. Thorpe (Manchester: Manchester University Press, 1973), pp. 13-32. 
Sador, ${ }^{21}$ et que Tristan lui-même ne participera à la Quête du Graal que pour mieux l'abandonner, de même Perceval est-il déconstruit d'emblée comme héros du Graal : désormais rejeton d'un lignage profane, dont il n'occupe pas la première place, ${ }^{22}$ ce fils d'un gardien du Graal selon Robert de Boron voit d'abord son destin borné par les passions humaines. On est bien dans cette perspective de 'laïcisation' du Graal qu'ont mise en évidence Emmanuèle Baumgartner et C.-A. Van Coolput.

\section{V.I, les ms BNF fr. 112-12599 et le cycle post-Vulgate}

Dans ces conditions, l'appartenance tristanienne des enfances de Perceval ne devrait pas faire de doute du point de vue esthétique.

Du point de vue philologique, le problème se pose en termes différents. Le récit consacré à Perceval se retrouve à l'identique, on le sait, dans une partie des ms BNF fr. 112 et 12599 qui donne une version particulière de la dernière folie de Lancelot et de sa quête par les

\section{${ }^{21}$ Cf. Emmanuèle Baumgartner, La Harpe et l'Épée. Tradition et renouvellement dans le} Tristan en prose (Paris: SEDES, 1990), p. 18 : 'Sador est aussi et surtout le héros à part, celui qui refuse de suivre ses frères [...]. À travers Sador, le héros non-conformiste, le texte du Tristan définit ainsi dès les premières lignes son double statut et son originalité : sa relation 'filiale' au Lancelot-Graal et son profil de fils prodigue qui refusera jusqu'au bout, jusqu'à la mort du héros, le retour sans condition dans la maison du père, l'alignement parfait sur le modèle'.

${ }^{22}$ Voir Champion II, § 42, où le prosateur explique que Perceval était moins bon chevalier que Lamorat : l'honneur du Graal 'ne li avint mie par la bonté de sa chevalerie conme il avint par la bonté de sa char', etc. Dans les lignes qui précèdent et suivent, le prosateur répète qu'il 'n'ert mie si bon chevalier conme Lamorat ses freres'. 
chevaliers, et que Fanni Bogdanow a identifiée comme une section du cycle post-Vulgate.

Cette Folie Lancelot inclut néanmoins les débuts de Perceval, précédés des aventures fatales de ses frères, au milieu d'autres aventures qui forment un ensemble plus complet (y compris pour Perceval lui-même, qui par exemple délivre le château de Beaurepaire, comme chez Chrétien). À la question de savoir si la partie Lamorat/Perceval de V.I est originelle ou non se superpose donc cette autre question : V.I a-t-elle prélevé les épisodes percevaliens qui l'intéressaient dans la plus vaste Folie Lancelot, qui donc serait sa source, ou bien au contraire la Folie Lancelot a-t-elle repris des épisodes de V.I pour ensuite développer les aventures d'autres chevaliers?

Les comparaisons menées par Fanni Bogdanow dans son édition de la Folie Lancelot sont à l'avantage chronologique de V.I : lorsqu'une divergence survient entre BNF fr. 757 (V.I) et 112-12599, l'éditrice montre que ce dernier ensemble a modifié sa source pour l'intégrer dans un corpus chevaleresque plus vaste, ou bien que la divergence est attribuable à une erreur commune que ne commet pas V.I (comme elle le développe dans les notes des chap. VII et VIII, et notamment p. 249). Aux nombreux éléments qu'elle mentionne, on peut même en ajouter. En voici deux exemples.

Tout d'abord, lorsque la mère de Perceval se plaint d'avoir perdu son mari et ses frères, le manuscrit BNF fr 112 abrège en particulier l'allusion à Merlin et à la Table Ronde, qui n'est pas explicitement mentionnée :

'Ha ! Dieux, fu oncques mais si maleuree dame ne si apouvrie et mise a mesaventure [tante] bonne dame et [tante] bonne damoyselle puis que tu fus encommencee ! Ha ! Merlin, tu la commenças, et par toy fu elle establie. Maldicte soit l'arme de toy, car se elle ne fust, je ne fusse ja tournee a si grant povreté ne a si grant doleur comme je suis, ne ja si haulte porteure 
comme Dieu m'avoit octroyee ne fust tournee a si grant douleur comme elle est.' (La Folie Lancelot, p. 85)

Dans cette version, le pronom elle semble renvoyer à la cour, dont l'établissement par Merlin est nettement moins satisfaisant que la leçon donnée par V.I, dans laquelle la mention de la Table Ronde rend l'adresse au devin plus claire :

'Ha ! cort de roi Artus, maldite soiez tu et confondue! A povreté et a deshonor te puisse ge encore veoir et revertire !Tu as apovroiee tantes bones dames et torné a mesaventure tantes puceles et tantes gentiles damoiseles qe jamés tant de bien ne feras conme tu as fet de mal ! 'Ha! Merlin, qui conmenças la Table Reonde et qui en porchaçast l'establiment, maldite soit l'ame de toi ! Se la Table Reonde ne fust, je ne fusse jamés tornee a si grant povreté [...].' (Champion II, p. 146).

Mais le plus notable est la formule par laquelle les manuscrits BNF fr. 112 et 12599 introduisent Perceval :

La teste de Lamorat remest leans en la court ne l'istoire ne devise pas que le roy en fist, ains s'en taist atant et retorne a compter comment Parceval ly virges vint a court, car de compter ceste branche [ne] nous povons nous souffrir que nostre livre n'en fust corrompuz. (La Folie Lancelot, p. 81)

Comme dans V. I, effectivement, après que la tête a été apportée à la cour et la valeur de Lamorat célébrée, le texte passe à autre chose. La formule de transition dans le ms BNF fr. 757 est : 'si en lesse orez atant li contes a parler et retorne a Agloval por deviser coment il 
mena son frere Perceval a la cort le roi Artus' (Champion II, p. 138). Le développement supplémentaire de la formule d'entrelacement de 112-12599 traduit certainement la surprise d'un copiste qui attendait la suite (que devient la tête ?), qui ne la trouve pas, qui suit sa source à contrecœur, sans comprendre sa logique et qui, pour masquer ce qui lui semble une incohérence, s'appuie sur le refus de corrompre l'histoire.

D'autres constats, cependant, tout aussi nombreux, imposent le point de vue contraire. Ils portent, en amont de l'apparition de Perceval, sur les aventures qui mènent à la mort de Lamorat et Driant ; en aval, sur la libération de Tristan par Perceval et le voyage du héros délivré jusqu'en Logres dans la Nef de Joie.

Pour les aventures antérieures, Emmanuèle Baumgartner avait déjà souligné qu'une allusion très explicite était faite à l'épisode de la libération de Gauvain du 'chastel as .X. chevaliers' par Lamorat (voir Essai d'interprétation, pp. 43-44), ce qui implique probablement que le prosateur connaît de cette aventure une relation détaillée. L'abrègement a manifestement laissé des traces :

Lamorat sanz faille l'en delivra. Mes se ne vos deviserons mie coment il le gita de ce chastel, car trop avons a deviser d'autres choses, ainz vos traiterons au plus tost qe nous porrons a l'estoire de Perceval, car par cele nos convient il a revenir a nostre estoire ou nous vos deviserons conment et en quel maniere misere Tristan fu delivré de son oncle. (Champion II, $\S 43)$

Car dans ces propos qui rappellent de près ceux que le prosateur de V.II tient lorsqu'il abrège V.I (cf. Droz III, $\S \S 165,187,198 \ldots$ ), le prosateur confesse qu'il n'est plus tout à fait dans 'nostre estoire' et que si Perceval permet de rejoindre cette dernière, c'est par le biais de 
la délivrance de Tristan -- péripétie absente de la Folie Lancelot.$^{23}$ On pourrait éventuellement arguer que la copie n'est pas l'œuvre, et que le copiste, qui travaille bien après la genèse du Tristan, peut par acquit de conscience mentionner d'autres versions qu'il connaît du texte qu'il recopie, éventuellement plus tardives. Cependant tous les témoins consultés pour l'édition s'accordent sur ce passage, ce qui prive une telle hypothèse de toute inférence vérifiable.

C'est surtout la partie postérieure aux aventures de Perceval qui jette le doute sur l'ensemble du récit propre à V.I. Le voyage des amants dans la Nef de Joie, après la délivrance de Tristan par Perceval et diverses péripéties en Cornouailles, apparaît tout à fait déplacé dans le Tristan. Des erreurs de ton ou des maladresses trahissent la réfection. Emmanuèle Baumgartner s'était déjà étonnée de l'entrevue morale avec le roi Pêcheur et des reproches d'Yseut à Tristan devant la fontaine empruntée au Chevalier au Lion (loc. cit., p. 45). Il est permis de se demander également, avec les éditeurs, pourquoi surgissent, si rapprochées dans le texte, deux nefs merveilleuses -- la Nef de Joie 'après celle de Perceval (§ 185), dans un roman qui les ignore presque totalement par ailleurs' (Champion II, p. 444, sur le § 136) -- ou d'où vient à Yseut non seulement qu'elle connaisse la Nef de Joie ou les motivations de Merlin, mais surtout qu'elle prophétise sur la fin du royaume d'Arthur. ${ }^{24}$ Mais

${ }^{23} \overline{\text { Voir cependant plus loin la n. } 44}$ et le paragraphe qui l'introduit.

${ }^{24}$ Champion II, $\S 138$ : 'Ceste [...] est la nef que Merlin fist fere pour la fille au roi de Norhombellande et por chacier le suen ennemi. [...] Je vous conterai mot a mot coment ele fu fete et par quel guise et coment ele perira et par quele aventure. Mes ce ne sera pas a vostre tens ne au mien, ce sera un poi aprés le departement que li rois Artus fera quant il se departira du tout du royaumee de Logrez aprés la doloreuse bataille qui sera faite en la plaingne de Salibieres, la ou tuit li prodoume de la Grant Bretaigne seront torné en un sol jor a duel et a 
plus que tout, pour la question qui nous occupe, le voyage des amants semble recomposé à partir de données éparses empruntées aux épisodes de 12599-112 que V.I ne donne pas, et dans lesquels Tristan remplace Érec ou Perceval (Emmanuèle Baumgartner, Essai d'interprétation, p. 46-47). Dans ces conditions, force est de conclure à l'antériorité de la Folie Lancelot sur le texte de V.I.

D'autre part, après Emmanuèle Baumgartner, de nombreux critiques ont infirmé plus ou moins directement l'idée héritée d'E. Löseth de la primauté chronologique de V.I, et notamment du ms BNF fr. 757. Alors que Fanni Bogdanow est demeurée attachée à cette opinion jusque dans son édition de la Queste et de la Mort Artu post-Vulgate, et pensait que ce manuscrit était le plus fidèle au Tristan originel qui nous soit demeuré, il apparaît en réalité qu'il est une composition relativement tardive, qui présente des traces d'abrègement et qui ne saurait donner à lire un récit antérieur au cycle post-Vulgate. ${ }^{25}$ Philippe Ménard a démontré il y a peu que le prosateur de la dernière partie de V.I, celle qui se déroule pendant la Quête du

destrucion.' La mention de la 'fille du roi de Northumberland' renvoie sans doute à la Suite du Merlin post-Vulgate, bien que la Nef de Joie n'y apparaisse pas.

${ }^{25}$ Sur le manuscrit BNF fr. 757, voir notamment J. Blanchard, éd., Le Roman de Tristan en prose. Les deux captivités de Tristan (Paris: Klincksieck, 1976), pp. 28-31 ; les cinq volumes de l'édition Champion ; ainsi que le récent article de Fabrizio Cigni, 'Per un riesame della tradizione del Tristan in prosa alla luce di vecchie e nuove edizioni, con nuove osservazioni sul ms. Paris, BNF, fr. 756-757', in Culture, livelli di cultura e ambienti nel Medioevo occidentale. Atti del IX Convegno della Società Italiana di Filologia Romanza (S.I.F.R.), éd. Fr. Benozzo et al. (Rome: Aracne, 2012), pp. 247-278. Dans le t. V de l'édition Champion, Christine Ferlampin-Acher établit que ' $N$ a procédé à un travail de réécriture, à partir d'une version plus ancienne, dont $A$ à ce moment serait plus proche' (p. 80) tandis que de son côté Fabrizio Cigni pointe également des indices de réfection. 
Graal, connaissait la dernière partie de la post-Vulgate : il fait référence explicitement à des épisodes qui pour leur majorité se retrouvent dans l'édition de Fanni Bogdanow, références unifiées par le renvoi à l'autorité de Robert de Boron. ${ }^{26}$ Avec la certitude que la partie Queste de V.I est postérieure à celle du cycle post-Vulgate, il semble délicat d'imaginer que la guerre des lignages mise par écrit dans V.I puisse prétendre à l'antériorité sur les pages équivalentes de la Folie Lancelot.

\section{Une génération composite}

Nous nous trouvons ainsi face à un faisceau d'indices contradictoires, qui font pencher légèrement la balance vers l'antériorité de 112-12599 sur V.I. Faut-il compléter ou corriger une partie des raisonnements avancés ou des données rappelées ci-dessus ? Ou bien peut-on imaginer un mode de transmission des textes qui explique ces contradictions ?

Nous proposons l'hypothèse selon laquelle la matière de ces épisodes percevaliens est bel et bien d'origine tristanienne, mais que la forme textuelle qui apparaît dans tous les manuscrits conservés est allogène, et issue du développement particulier appelé la Folie Lancelot, qui est probablement une partie du cycle post-Vulgate.

En d'autres termes, une version préalable du Tristan contenait des pages consacrées à la rivalité des lignages de Lot et de Pellinor, et préparait bien à l'apparition du rôle de Perceval, libérateur de Tristan. Le Tristan n'a en rien besoin de la post-Vulgate pour établir un tel scénario. En premier lieu, il faut bien qu'il y ait eu un emprisonnement de Tristan et sa

${ }^{26}$ Philippe Ménard, “"Monseigneur Robert de Boron" dans le Tristan en prose', in Des Tristan en vers au Tristan en prose. Hommage à Emmanuèle Baumgartner, éd. L. HarfLancner, L. Mathey-Maille, B. Milland-Bove et M. Szkilnik (Paris: Champion, 2009), pp. 359-370. 
délivrance, suivie d'un retour en Logres -- postulat infiniment moins gratuit et plus praticable que l'idée contraire. Or rien ne vient infirmer le rôle pressenti de Perceval comme libérateur, ce qui laisse la place à une section du récit qui devait l'introduire au milieu des désordres de la cour, tous points sur lesquels V.II confirme la version de V.I.

Comme l'a remarqué avec fermeté Gilles Roussineau, la guerre des lignages de Lot et de Pellinor n'est pas dans le récit tristanien une importation postérieure au cycle postVulgate : elle fait 'partie de la trame originelle du Tristan en prose, ${ }^{27}$ car elle apparaît lors du passage de Tristan dans la forêt de Darnantes, dans le premier quart du roman, lorsque Tristan se rend en Cornouailles avec Kahédin : c'est là que Gauvain exprime pour la première fois sa haine pour Lamorat (éd. Curtis, t. III, § 794). Il serait si simple que cet épisode, absent de quelques manuscrits, soit une addition... Cependant, on a montré que les témoins qui ne le reproduisaient pas y faisaient allusion assez ouvertement, si bien qu'il ne peut être exclu de la 'trame originelle' du Tristan (voir par exemple Emmanuèle Baumgartner, Essai d'interprétation, p. 37). C'est la preuve que la haine des deux lignages n'est pas un thème adventice.

Lamorat lui-même est, un peu plus tôt dans le récit, le héros malheureux d'aventures au Val de Servage. Celles-ci sont, quant à elles, une addition très probable, mais également très précoce, d'après Renée Curtis (éd. cit., t. III, p. XXV); ces épisodes sont connus et exploités par le Guiron, notamment). Ces aventures sont pourtant précédées d'une brève entrevue entre Lamorat et Driant d'une part, Tristan et Yseut de l'autre, et rien ne conduit à penser que cet épisode-ci, représenté dans toutes les familles de manuscrits, et dans lequel

\footnotetext{
${ }^{27}$ G. Roussineau, 'Remarques sur les rapports entre la Suite du Roman de Merlin et sa continuation et le Tristan en prose', in Miscellanea Medicevalia, Mélanges Philippe Ménard, (Paris: Champion, 1998), t. II, pp. 1149-1162 (n. 76).
} 
Lamorat aime déjà la reine d'Orcanie, soit étranger à un état premier du roman. ${ }^{28} \mathrm{Il}$ est donc probable que le Tristan invente le personnage et son amour pour la mère de Gauvain.

Pour le rôle de Perceval, il suffit très certainement de considérer que la 'délivrance,' par ce dernier, de Lancelot dans l'île de Joie a pu naturellement servir de modèle au Tristan, et ce d'autant que Lancelot était déjà prévu comme le premier libérateur de Tristan -- une allusion précise à une discussion qu'il a avec Marc lors de cette épisode se trouve très au début du roman, lors du meurtre de Pernehan : Et puis le reprocha Lancelot au roi Marc, si com nostre estoire le devisera apertement (éd. Curtis, t. I, § 243). Du reste, dans le Lancelot, la première aventure de Perceval aboutit également à une délivrance, celle de Persidès (voir ci-dessus n. 18) : c'est une deuxième raison pour que le rôle de Perceval se cristallise dans cette fonction de libérateur pour l'auteur du Tristan.

Quant à l'idée d'une guerre des lignages, elle est simplement empruntée à la Mort Artu, qui, rappelons-le, a laissé une empreinte notable sur la partie du roman considérée. Il est assez naturel que ce thème se soit porté sur Lamorat. Il fallait un autre lignage que celui de

${ }^{28}$ Voir éd. Curtis, t. II, p. 29. Soulignons qu'il figure donc dans les ms d'Aberystwyth (NLW 446) et de Modène, qui sont justement les deux seuls qui aient supprimé les aventures ultérieures de Lamorat et Brunor. Il faut noter que la première rencontre entre Lamorat et Gauvain se déroule pendant la partie probablement rajoutée qui précède les aventures du Chevalier à la Cotte Mal Taillée, et qu'en dépit de l'hostilité entre les deux personnages, aucune mention n'est faite du problème lignager (éd. cit., § 626). En revanche, Lamorat venait de rencontrer Frolle, qui indique dans la plupart des manuscrits que Lamorat a tué son père, mais surtout, dans les ms BNF fr. 750 et 12599 (qui semblent donner des versions plus anciennes des aventures de Lamorat, cf. ibid. p. 47), que le père de Lamorat a tué le sien. La proximité (quelques lignes) entre ce thème et l'apparition de Gauvain sur le chemin de Lamorat est troublante. 
Lancelot, pour ne pas anticiper sur la Mort Artu, et cela ne pouvait être celui de Tristan, héros solitaire. Lamorat est un des doubles de Tristan que met en scène le roman, ou à tout le moins l'un de ses séides (comme le montre Christine Ferlampin-Acher, Champion V, p. 88). Son nom laisse entendre mort et amor et préfigure ses amours tragiques, une destinée éminemment tristanienne. Ce champion de la chevalerie inventé par l'auteur, avec la sympathie qu'il suscite, était tout trouvé pour illustrer la dégradation des relations chevaleresques et surtout le noircissement du lignage de Gauvain. En outre Lamorat de Gales s'apparente facilement et habilement à Perceval le Galois : en mélangeant le souvenir de la bipartition Perceval/Gauvain du Conte du Graal et l'opposition entre Gauvain et les élus du Graal dans la Queste, ${ }^{29}$ l'auteur constituait à peu de frais le lignage tout à fait prestigieux de Pellinor, rival considérable, et donc cible privilégiée, de celui d'Orcanie, celui-là même dont Richard Trachsler a montré la propension, dans le Tristan, à s'attaquer aux représentants nouveaux de la chevalerie arthurienne. ${ }^{30}$ De ce point de vue encore, Lamorat et ses frères s'intègrent dans l'organisation générale (narrative et intertextuelle) du Tristan.

${ }^{29}$ Le nom de Pellinor n'est pas étranger au lignage du Graal et a pu être confondu avec Pellès ou Pellehan. Voir les articles Pellinor ${ }^{1}$ et Pellinor ${ }^{2}$ dans Gerald D. West, An Index of Proper Names in French Arthurien Prose Romances (Toronto: University of Toronto Press, 1977). ${ }^{30}$ Richard Trachsler, Clôtures du cycle arthurien, Publications Romanes et Françaises, 215 (Genève: Droz, 1996), p. 180. Rappelons que la méchanceté de Mordret est issue de la préhistoire des textes arthuriens, que celle d'Agravain 'l'Orgueilleux' est déjà montrée par le Lancelot. Le noircissement de Gauvain dans le Tristan n'est pas tributaire du cycle postvulgate : il conjoint la sécheresse morale dont l'accuse la Queste et la démesure guerrière qu'il manifeste dans la Mort Artu. Le Tristan y fait allusion dès les premières aventures du héros : 'Et neporquant, je sai bien que messire Gauvens est plains de felonie coverte.' (éd. Curtis, t. I, § 417). Rien n'indique qu'il puisse s'agir d'une interpolation, d'après l'accord 
Tout cela laisse entendre que le scénario d'un éventuel Tristan antérieur au cycle postVulgate ne devait pas être bien différent, concernant cette partie du récit, de ce que nous montrent les témoins qui nous sont demeurés. Il reste que les références que fait V.I à la Folie Lancelot semblent décisives pour donner la priorité au texte de cette dernière sur celui du Tristan de V.I. De l'autre côté, les indices microtextuels mis en valeur par Fanni Bogdanow (ou par nous-mêmes quelques paragraphes plus haut), qui indiquent le contraire, peuvent aussi bien s'expliquer par une source intermédiaire. V.I aurait certes recopié un texte légèrement différent de 112-12599 et plus proche de 1'،original' commun ; mais cet 'original' pourrait avoir été une version déjà considérablement modifiée d'une forme primitive du récit inclus dans le Tristan, forme perdue et différente de ce que présente V.I.

Un processus somme toute très simple expliquerait aisément cette situation : à partir d'une version initiale de la rivalité des lignages et des enfances de Perceval donnée par une forme antérieure du Tristan, un développement relativement indépendant leur a été consacré $;{ }^{31}$ un copiste ultérieur du Tristan, retrouvant là ce qu'il estimait assez légitimement

général des manuscrits consultés. De ceux de Paris, d'Edinburgh et de Genève qui donnent correctement cette partie du roman, seule une petite minorité ne présente pas ce jugement sur Gauvain : BNF fr. 758, Edinburgh Ad. 19.1.3, qui abrègent ou réécrivent volontiers dans le détail ; ainsi que le ms Bodmer 164.

${ }^{31}$ Notons que dans le manuscrit BNF fr. 12599, cette suite d'aventures, qui se trouve donc dans seulement deux manuscrits, fait pendant à des péripéties censées se dérouler peu avant et pendant la Quête du Graal, et inconnues dans tous les autres témoins. Ces deux passages si spécifiques constituent le troisième fragment dont le recueil est composé. On peut les considérer comme une unité codicologique ou un élément codicologique. Sur la lisibilité de l'appartenance de ce texte au cycle post-Vulgate, voir les dernières pages de cet article. 
appartenir à son texte, mais sous une forme plus développée, donc pour lui plus satisfaisante, a souhaité composer avec les chapitres concernés de la version refaite. ${ }^{32}$ Le résultat de ce travail a remplacé unanimement, dans les témoins de V.I, l'organisation originelle.

Bien que celle-ci soit à jamais inconnaissable, nous pouvons essayer de détailler autant que possible la marche qu'aurait suivie ce remanieur, l'auteur du modèle de V.I.

Il est difficile de dire si les épisodes de V.I qui ne figurent pas dans la Folie Lancelot sont d'origine ou ont été ajoutés au même moment : une étude de détail de chacun d'entre eux dépasserait largement le cadre de cet article, pour des gains incertains. Ainsi de la version très différente que donnent V.I et la post-Vulgate des premières aventures de Gaheriet : Brunor y a-t-il été réintroduit par V.I, ou bien la post-Vulgate a-t-elle escamoté Brunor, personnage qui ne l'intéresse pas? Il n'est guère possible de répondre, même s'il est manifeste que la querelle interne au lignage de Gauvain (Gauvain et Agravain contre Gaheriet) a peu mobilisé l'attention du Tristan, occupé à mettre en place la rivalité entre lignages différents. ${ }^{33}$ Érec, quant à lui, devait être absent des aventures originales, et c'est bien parce que le prosateur de V.I ne savait que faire de lui qu'il a dû abréger l'épisode du Château des Dix

${ }^{32}$ Il est acquis que ni BNF fr. 112 ni BNF fr. 12599 ne peuvent être eux-mêmes la source de V.I. Il est donc indifférent de supposer que V.I travaillait avec une Folie Lancelot plus proche de son original, qui expliquerait les meilleures leçon conservées dans le détail du texte selon Fanni Bogdanow.

${ }^{33}$ Le Tristan réserve une place très favorable à Gaheriet, premier témoin de la valeur de Tristan et compagnon d'armes au tournoi de Louveserp, alors que dans le même temps Gauvain est tellement noirci. Une querelle interne au lignage d'Orcanie n'est pas hors de propos dans le Tristan, mais peut aussi apparaître comme l'excroissance ultérieure d'une thématique alors en germe. Dans V.I, Gaheriet préfère renverser Gauvain pour soutenir Hector plutôt que d'épargner son frère (Champion I, p. 328). 
Chevaliers, qui concerne pourtant Gauvain et Lamorat. Il est remarquable que, dans la Folie Lancelot, la haine initiale éprouvée par Gauvain et ses frères envers le lignage de Lamorat se dilue assez rapidement, et le récit se décentre sur Érec.

Pour cette dernière raison, on incline à penser que les joutes de Perceval contre Keu, Gauvain et Mordret faisaient partie de l'original tristanien, plus fermement orienté vers la querelle des deux lignages, dans la mesure où les menaces autrefois proférées envers Lamorat se renouvellent à présent contre Perceval. Bien sûr, une prudence extrême s'impose. Rappelons que l'accord de V.I et 112-12599 est brisé ici et que l'on trouve dans la Folie Lancelot, à la place de l'épisode des gouttes de sang, celui de Perceval et Gaheriet dans l'île de la sœur de Perceval. Comme l'a bien vu Emmanuèle Baumgartner, V.I a déplacé ailleurs cette aventure, attribuée à présent à Tristan et Lamorat, qui rappelle Méraugis de Portlesguez de Raoul de Houdenc (Essai d'interprétation, p. 47). Rien n'interdit de penser que V.I a simplement rajouté au texte de la Folie Lancelot qu'il recopiait l'épisode des gouttes de sang sur la neige, après avoir déplacé le premier épisode. Mais le recours à Chrétien de Troyes est encore très proche ici de celui qui caractérisait la rencontre de Perceval et Agloval : appelé naturellement par l'intertextualité liée au personnage de Perceval, il demeure sobrement adapté au déroulement du récit et à ses préoccupations et semble relever du même mouvement d'écriture que le précédent épisode.

En revanche, la partie qui narre la délivrance de Tristan et la navigation des amants jusqu'en Logres procède, elle, d'un mouvement d'écriture différent. Recomposée à partir des aventures de la Folie Lancelot que les chapitres précédents n'avaient pas intégrées, elle a dû constituer pour son auteur à la fois le moyen de fondre ensemble, au bénéfice de Tristan, les 
péripéties qu'il trouvait dans la Folie Lancelot mais pas dans le Tristan ${ }^{34}$ et l'occasion de se livrer à ce qui nous apparaît comme un exercice d'écriture, une fantaisie sur des thèmes de Chrétien de Troyes. Cela explique l'aventure de la fontaine du Pin, seule péripétie du lot qui n'ait pas sa racine dans la Folie Lancelot. Cette fois, cependant, le recours à l'inventeur du genre ne s'occupe guère de s'adapter au récit-cadre : nous en rappelions plus haut les discordances manifestes et les gratuités contradictoires. Qu'elle appartienne ou non à la même strate de réécriture, cette section repose quoi qu'il en soit sur un parti esthétique bien différent de celles qui précèdent. On peut en épier les particularités ${ }^{35}$ mais il semble clair qu'elle est complètement adventice dans le Tristan.

On peut ainsi résumer le processus impliqué par notre hypothèse : l'auteur de V.I, recopiant le Tristan mais ayant à sa disposition une Folie Lancelot, a réadapté selon cette dernière le texte originel du Tristan, désormais perdu pour nous.

${ }^{34}$ En témoignent la transposition du rôle de Mabon/Nabon et le passage devant le Roi Pêcheur ; cf. éd. Bogdanow, pp. 108, 111, 152-153, et Emmanuèle Baumgartner, Essai d'interprétation, p. 46-47.

${ }^{35}$ La réécriture de Chrétien dans l'épisode de la Fontaine au Pin a été étudiée de près (Annie Combes, 'Une écriture de la dissemblance : la Fontaine au Pin dans le Tristan en prose', Des Tristan en vers au Tristan en prose, pp. 103-117). Sur la position particulière de la navigation des amants dans le récit tristanien, voir Bénédicte Milland-Bove, 'Des Tristan en vers au Tristan en prose ? Métamorphose du voyage maritime des amants dans les deux versions du Tristan en prose'., ibid., pp. 87-101, et D. de Carné, 'Dynamique d’une géographie romanesque : les espaces des matières narratives dans le Tristan en prose', Espaces et mondes au Moyen Âge, éd. Mihaela Voicu (Bucarest: Presses de l'Université de Bucarest, 2009), pp. 92-101. 
Les aventures communes aux deux textes figurent dans V.I sous une forme proche de la Folie Lancelot, sans que l'on puisse déterminer si cette proximité provient seulement du recopiage de la Folie Lancelot par V.I, ou bien remonte à l'original tristanien commun.

Les autres épisodes de la Folie Lancelot n’ont pas été repris probablement parce que leur matière ne rencontrait pas celle du Tristan que l'on recopiait.

On ne peut rien dire sur les épisodes propres à V.I, encore qu'il semble probable que l'épisode des gouttes de sang soit d'origine dans le Tristan, car le traitement des sources y est beaucoup plus proche de l'épisode antérieur de la rencontre entre Perceval et son frère que de la section subséquente de la navigation des amants.

Cette dernière est une réfection évidente, qui fait apparaître clairement que la Folie Lancelot était dans son entier connue de V.I. Elle nous montre peut-être aussi que la version primitive de la libération et du voyage du protagoniste devait être assez sommaire, puisqu'elle n'a pu résister à cette réécriture sous aucune forme dans la tradition manuscrite -- V.II laisse ici un blanc --, et qu'elle reste un point de fracture important au sein de celle-ci, tout autant qu'un lieu du récit propice aux renouvellements : la version V.IV proposera une refonte complète de ces épisodes que l'auteur de V.II jugeait déjà trop peu satisfaisants pour les intégrer (consulter le résumé du ms BNF fr. 99 dans l'analyse de Löseth, à partir de $§ 282$ e, ainsi que ses remarques sur BNF fr. 103).

La présentation de cette hypothèse, qui peut légitimement susciter le désaccord, appelle quelques précisions d'ordre plus général sur la Folie Lancelot, la post-Vulgate et sur le mode de composition des romans en prose ainsi entendu.

Concernant le mouvement de va et vient des épisodes d'un texte à l'autre qui apparaît dans notre hypothèse, et la distinction matière/texte qui l'accompagne, on peut trouver qu'elle 
n'est qu'une solution médiane, voire de facilité, et la juger inutilement complexe. Elle apparaît cependant comme la plus à même de concilier les contradictions factuelles auxquelles se heurte la réflexion lorsque l'on souhaite déterminer la primauté chronologique de l'un et de l'autre. Du reste, elle n'est pas une solution ad hoc, bâtie pour un problème unique, et dont le processus serait sans exemple. Dans les rapports entre le Tristan et la postVulgate, la partie Queste laisse voir des phénomènes qui s'expliquent parfaitement par une telle hypothèse, et que l'on peut mettre clairement en lumière par un exemple éloquent. Au t. VIII de l'édition de V.II, la querelle entre Tristan et Palamède aboutit à la mort accidentelle d'Habé le Renommé, pris par Tristan pour Palamède (§§ 141-142). Sur ce dernier, le narrateur fait au début de l'épisode cette étrange remarque :

Et saciés que aventure l'ot amené cele semainne devant la Joieuse Garde, droit a la porte u il avoit veüe la roïne Yseut. Et tantost com il le vit, pour la grant biauté qui en li estoit, l'ama il adont de si grant amour qu'il n'amoit autretant soi ne autrui. Ne nus ne le met hors de cel penser qu'il ni peüst avenir en aucune maniere a l'amour de li, mais qu'il savoit bien par le tesmoing de gens que mesire Tristrans estoit uns des biaus cevaliers du monde et des meilleurs. (Droz VIII, § 140)

Si distrait qu'il se soit montré, un lecteur du Tristan trouvera impensable que Palamède soit tombé amoureux d'Yseut la semaine passée (nous sommes à l'avant-dernier tome du roman : Palamède aime Yseut, qu'il a rencontrée en Irlande et non à la Joyeuse Garde, depuis une vingtaine d'années) et que Tristan soit encore pour lui un inconnu dont il ne connaisse la beauté que par ouï-dire (ils se sont souvent rencontrés, battus l'un contre l'autre, et accompagnés l'un l'autre). Des erreurs de détail existent dans le Tristan, mais une si fondamentale distorsion du scénario d'ensemble ! Aussi Fanni Bogdanow a-t-elle beau jeu 
d'inclure cet exemple dans la liste des contradictions qui doivent selon elle prouver que le Tristan recopie la Queste post-Vulgate. ${ }^{36}$ Soit, mais la mort d'Habé est annoncée dès le premier séjour de Tristan en Irlande, dans des termes qui correspondent exactement à l'action racontée, et dans une partie du récit dont l'ancienneté et l'authenticité sont difficilement soupçonnables :

Et a la queste del Saint Gaal le fist il mout bien. Mes illec l'ocist Tristanz par grant mescheance, a celi point qu'il sivoit Palamedes, le bon chevalier sarrazin, qui tant amoit ma dame Yselt. Et se aucuns me demandoit coment il avoit non, je diroie qu'il avoit non Hebés ; et por la proesce de li fu il apelez Hebés li Renomez. (éd. Curtis, t. I, § 323$)^{37}$

Si bien que l'on s'accorde facilement à ce que le texte reproduit par V.II soit allogène (quel copiste ayant ne serait-ce qu'entendu résumer le Tristan pourrait commettre pareille bourde ?), mais que la matière de l'épisode est proprement tristanienne, et prévue de longue date par le(s) prosateur(s) $:^{38}$ indépendamment de sa forme d'existence préalable (une

\footnotetext{
${ }^{36}$ Fanni Bogdanow, 'Un nouvel examen des rapports entre la Queste post-Vulgate et la Queste incorporée dans la deuxième version du Tristan en prose', in Romania, 118 (2000), pp. 1-32, et son édition de la Queste post-Vulgate, t. I, p. 83.
}

${ }^{37}$ Là encore, aucune variante significative n'apparaît dans les ms cités n. XXXXX, si ce n'est le ms fr. 189 de la Bibliothèque de Genève (annonce la péripétie mais sans le nom d'Habé), le ms Bodmer 164 (pas de mention de la Quête du Graal), le texte trop résumé de Paris, BNF fr. 758. L'annonce est également totalement absente du ms d'Edinburgh.

${ }^{38}$ Nous ne souscrivons pas à l'idée selon laquelle un premier Tristan ne mêlait pas son héros au monde arthurien. Considérer que son substrat primitif est 'assez facile à isoler' (Emmanuèle Baumgartner, Essai d'interprétation, p. 92) est faire peu de cas de l'incrustation très précise de la comparaison à Lancelot au sein du parcours de Tristan, et ce dès l'origine. 
première version, ensuite remplacée ? une première version identique à laquelle un scribe zélé a rajouté cette maladroite précision ? ou bien pas de version originelle, le texte étant le pur produit de la post-Vulgate, mais commandé par les indications du Tristan ?), la mort d'Habé le Renommé était censée appartenir au Tristan. Un prosateur du Tristan était fondé à se réapproprier cet épisode s'il le trouvait ailleurs -- aurait-il même eu conscience que le texte où il le trouvait n'était pas un Tristan ? Et l'on voit réapparaître dans cet exemple la disjonction entre matière romanesque et forme textuelle, question qui interroge bien plus l'identité propre des récits et la perception de cette identité par les lecteurs et les copistes, que des phénomènes plus étroitement philologiques.

On ne voit pas comment isoler une couche arthurienne d'une couche plus primitive sans tomber dans un exercice tout à fait artificiel. Par ailleurs le prologue de Luce et la première apparition de Merlin indiquent qu'il faut attendre une quête du Graal dans la destinée du héros, héros déjà mis sur un pied d'égalité avec Lancelot et Perceval (éd. Curtis, t. I, prologue et $\S 236$ ). En outre, ce que Guiron et la post-Vulgate connaissent du Tristan (donc à des dates supposées antérieures à V.II et à V.I) montre que, contrairement à l'avis d'Emmanuèle Baumgartner et de Renée Curtis, le Tristan d'avant 1240 ne devait pas être si fondamentalement différent de celui/ceux que nous connaissons nous-mêmes. Voici une liste non exhaustive d'éléments qui devaient s’y trouver, parfois très avancés dans le cours du récit : la dégradation morale et chevaleresque de Gauvain, le combat du Perron Merlin (prédit dans la Suite du Merlin), la guerre contre les Saxons (reprise avec de nombreux points communs dans Guiron), l'entrevue houleuse entre Lancelot et Marc (et donc la délivrance de Tristan par celui-là), le Val de Servage, l'existence de Lamorat et sa rivalité avec Gauvain, la présence de Brunor et de Dinadan, tous deux frères (ce que sait le Guiron), la quête du Graal, enfin la mort des amants, puisque Marc apparaît inopinément comme un constructeur de tombeaux dans la Suite du Merlin. 
Plus largement, l'idée que des échanges aient lieu entre différents romans au cours même de leur genèse a déjà été avancée pour les ensembles en prose. C'est la perspective à laquelle aboutissait la réflexion de Gilles Roussineau sur un aspect du problème débattu ici. ${ }^{39}$ Emmanuèle Baumgartner envisageait également le rapport entre ces textes d'un point de vue plus dynamique que strictement chronologique :

Mais plutôt que de chercher à fixer les rapports chronologiques des deux textes, ne serait-il pas plus judicieux d'étudier le jeu des anamorphoses auxquelles se livrent leurs rédacteurs, de s'interroger sur le sens de la notion d' 'auteur,' s'agissant de compilations aux contours si mouvants $[\ldots] ?^{40}$

Récemment, Carol Chase a montré que l'idée d'une composition conjointe était la manière la plus efficace d'expliquer les influences réciproques et contradictoires que

${ }^{39}$ G. Roussineau, 'Remarques....', p. 1162 : 'Le Tristan et la Suite ont donc été rédigées dans la même période, entre 1230-1235 environ et 1240. Durant ce laps de temps, les échanges entre les copistes des deux œuvres, qui étaient presque contemporaines, ont dû être facilités et l'on peut raisonnablement présumer que les interpolations dont la version V. I. porte la trace ont été pratiquées à date ancienne'.

${ }^{40}$ Compte-rendu par Emmanuèle Baumgartner de l'article de Fanni Bogdanow, 'Un nouvel examen', dans Bulletin bibliographique de la Société Internationale Arthurienne, 53 (2001), p. 101. 
s'imposaient l'une à l'autre la Queste et l'Estoire del Saint Graal -- un problème assez proche de celui que nous rencontrons. ${ }^{41}$

Il faut ajouter que, bien qu'il pose au critique moderne, qui aimerait que les choses soient plus claires, des problèmes renouvelés, le recours à l'hypothèse d'échanges (sinon le constat qu'ils aient existé ?) rend certainement meilleure justice à la vie qu'ont pu réellement connaître les longues proses : non seulement parce qu'elles sont des travaux d'équipe, d'atelier, mais surtout du fait du temps d'élaboration très étendu du texte en prose et du livre qui le contient. ${ }^{42}$ Partant, il est plus que probable que les textes aient coexisté dans un état inachevé ou incomplet (on sait qu'ils voyageaient sous forme de cahiers), que des scénarios aient pu se voir remodelés en cours de composition (en fonction notamment de ce que produisait la concurrence), que des parties entières de textes aient pu s'amalgamer ou se désolidariser avant même que le(s) roman(s) qui devai(en)t les contenir ne soi(en)t terminé(s). Le texte envisagé comme une totalité finie, la perception des romans comme des blocs achevés avant de circuler, prenant place dans une succession ordonnée au sein de laquelle aucun n'empiète sur l'autre, empêchent dans certains cas de considérer dans une perspective adéquate la question de savoir si le roman A vient 'avant' ou 'après' le roman B.

Pour le Tristan, on peut remarquer qu'une première partie du récit jouit d'une grande stabilité dans la tradition manuscrite, que les divergences importantes se multiplient dans une

${ }^{41}$ C. Chase, 'La fabrication du cycle du Lancelot-Graal', Bulletin bibliographique de la Société Internationale Arthurienne, 61 (2009), pp. 261-280.

${ }^{42}$ Rappelons que le pseudo-Hélye indique dans l'épilogue de nombre de manuscrits du Tristan qu'il a mis cinq ans à composer son livre. On estimait encore récemment que le Tristan, la post-Vulgate et le Guiron devaient tous avoir été composés entre 1225 et 1235 ; comment concilier cet écart resserré, de si longues compositions et l'assimilation des textes les uns par les autres? 
partie médiane, et que dans la dernière partie, consacrée à la quête du Graal, la variance et surtout l'unité du roman deviennent quasiment impossibles à maîtriser. Cela suggère que le Tristan en prose a connu une composition très progressive, confrontée à mesure qu'elle avançait à un environnement romanesque changeant auquel les différentes versions ont réagi par des comportements dissemblables, et qui ne s'est pas appuyée sur les mêmes sources, les mêmes techniques et les mêmes préoccupations d'une manière égale d'un bout à l'autre du texte. $^{43}$

Enfin, concernant la Folie Lancelot, il n'est pas certain que l'auteur de V.I avait conscience d'avoir sous les yeux un texte 'post-Vulgate'. Si le texte conservé par les ms BNF fr. 112 et 12599 mentionne bien Robert de Boron (voir l'index de l'édition, p. 283), le prosateur de V.I n'y renvoie pas au moment d'abréger le passage de Gauvain au Château des Dix Chevaliers, contrairement à ce qui apparaît dans la partie consacrée à la quête du Graal. Par ailleurs, il convient de se demander au sein de quel ensemble textuel cette partie de la post-Vulgate s'offrait à la lecture. Elle n'est demeurée pour nous qu'insérée dans un manuscrit qui est une compilation arthurienne (le BNF fr. 112) et d'un autre qui, bien qu'étant une ébauche de compilation, est un manuscrit du Tristan (le BNF fr. 12599). Il est permis, sinon de douter que ce texte ait jamais existé sous une forme plus indépendante, à tout le moins de douter que le prosateur de V.I ait eu accès à un texte qui soit pour lui autre chose qu'un développement latéral d'aventures qu'il connaissait pour appartenir au Lancelot et au

${ }^{43}$ D. de Carné, Sur l'organisation $d u$ Tristan, indique que l'entrelacement se reconfigure complètement au fil des trois parties qu'il distingue dans V.II. Examinant les lieux d'exécution probables de presque tous les manuscrits du Tristan, Fabrizio Cigni ('Per un riesame', art. cit.), suggère que sa composition a pu suivre la progression géographique du texte. 
Tristan. La Folie Lancelot est loin de combler le manque qui sépare la fin de la Suite $d u$ Merlin que nous connaissons et le début de la Queste, non seulement en termes chronologiques, mais également du point de vue thématique : où est le personnage de Tristan, pourtant présent pendant la quête du Graal, annoncé dans la Suite du Merlin par la présence de Marc et celle du Morholt, et dont l'adjonction aux données issues du Lancelot est précisément le projet du cycle post-Vulgate (Fanni Bogdanow, La Queste Post-Vulgate, t. 1, pp. 36-37) ? Le ms BNF fr. 112 donne, entre les chapitres XIII et XIV de l'édition de Fanni Bogdanow, la délivrance de Tristan par Perceval. En présentant et en numérotant ainsi les chapitres, l'éditrice oblitère arbitrairement ce passage (elle va jusqu'à induire des formules d'entrelacement pour conjoindre les chapitres séparés par le matériau tristanien dans le manuscrit, qui, rappelons-le, est ici l'unique source matérielle). Or, en écrivant que 'there is no reason to assume that these incidents would have figured in the Roman du Graal, and they have therefore not been included in the present edition' (p. 273), elle évacue selon nous une question primordiale pour la définition de la Folie Lancelot : son modèle ne contenait-il pas justement ces épisodes tristaniens ? On pourrait, avec l'appui de la seule source manuscrite qui nous reste, retourner l'affirmation : il n'y a pas de raison d'envisager que ces péripéties n'aient pas figuré dans le Roman du Graal (i. e. la post-Vulgate). Ne retrouverait-on pas, dès lors, l'impression que l'ensemble identifié comme la Folie Lancelot est primitivement un développement conçu certes à part de la tradition existante, mais dans un contexte tristanien $?^{44}$

${ }^{44}$ Cela expliquerait deux bizarreries : 1. -- Dans V. I, la dame des pensées de Perceval, nommée Blanchefleur chez Chrétien, est devenue Elaine ; dans le BNF fr. 112, dans la reprise de Beaurepaire, la maîtresse des lieux reste anonyme. Cette réticence à suivre Chrétien sur l'identité de la jeune femme peut s'expliquer si l'épisode naît dans un contexte tristanien. En effet, dans les versions allemandes de Tristan et dans le Sir Tristrem anglais, la mère de 
Tristan est nommée Blanchefleur : même si ce nom n'apparaît pas dans le Tristan en prose, on peut le supposer connu. Le Tristan en prose, en renommant Heliabel la mère de Tristan, évite toute confusion avec le souvenir du Conte du Graal. L'anonymat de Blanchefleur durant le siège de Beaurepaire dans BNF fr 112, et son 'remplacement' par Elaine dans V.I, pourraient s'expliquer de la même façon : dans un récit consacré à Tristan, l'auteur ne peut nommer la dame de Perceval Blanchefleur. En revanche, dans un contexte comparable à celui de la post-Vulgate reconstituée par Fanni Bogdanow, quelle importance cela peut-il avoir ? Tristan n'est qu'un personnage secondaire, l'auteur n'est pas plongé dans un monde tristanien, il n'a pas de raison, alors même qu'il reprend les noms d'Aguigeron et Clamadam d'éviter celui de Blanchefleur. Il était logique que l'auteur de l'épisode ne nomme pas celle qui est dans la tradition l'amie de Perceval : plus tôt dans le Tristan, elle est devenue Elaine, et Chrétien, qu'il récrit alors, la nommait Blanchefleur : l'anonymat reste prudent. 2. -- Le problème chronologique qui concerne V.I et la Post-Vulgate implique également V.II. Les épisodes donnés au début de l'édition Bogdanow (le tournoi remporté par Lamorat, le meurtre de la reine d'Orcanie par Gaheriet) sont absents de V.I mais donnés par le Tristan de V.II. L'éditrice indique qu'il n'est pas possible d'induire une chronologie de la comparaison des textes de V.II et de BNF fr. 112 (12599 fait ici défaut), mais que V.II 'appears truncated' (p. 218) ; il faut cependant se défier de la cohérence acquise dans les textes plus récents qui peut donner l'illusion de la primauté sur les textes plus anciens. Quoi qu'il en soit, le ms 112 présente au début de la Folie Lancelot les fils de Pellinor comme des inconnus ('Et sans faille du roy Pellinor estoient issus cinq filz et une fille. Les .IIII. estoient a celui temps chevaliers, le quint jeune enfant. Et estoit ly ungs appellés Tor, ly autres Agloval, ly autres Drians, le quars Lamorat et le quint, qui encores n'estoit pas chevalier, avoit a nom Perceval. Et sachés que le plus preux qui fust de tous les freres fut Lamorat, et estoit de son aage longs et graisles et trop bel chevalier durement', etc., p. 1), présentation normale dans le cadre du Tristan, mais 


\section{Conclusion}

Les enfances de Perceval sont une très intéressante manifestation de la manière dont le Tristan se réapproprie ses sources. Dans un passage marqué par une divergence fondamentale de la tradition manuscrite (et par l'emprisonnement de Tristan, qui laisse le champ libre à des héros plus ou moins confirmés), l'intégration de Perceval à la trame du récit entrecroise diverses préoccupations spécifiques à l'entreprise du Tristan en prose. L'inclusion du personnage à un système de lignages antagonistes participe de la vision résignée de la chevalerie qui, dans le Tristan, prévient et prépare la Mort le roi Artu : dissensions internes, limites ou refus des valeurs chevaleresques précipiteront le monde arthurien dans la ruine comme elles ont entraîné le destin fatal des frères de Perceval. Le Graal, dans le Tristan, n’est qu'un catalyseur de malheurs à venir de la société arthurienne, et il est manifeste que la reprise conjointe de Chrétien de Troyes et du Lancelot en prose prive Perceval de toute transcendance, à part quelques reliquats de surface. On peut douter de ce que tout le passage, une grande partie du t. II de l'édition de V.I, soit une interpolation de la post-Vulgate. Quand bien même ce serait le cas, l'intégration de Perceval dans le Tristan trouve sans peine sa justification esthétique si elle est rapportée aux vastes enjeux du roman. Mais le problème philologique qu'elle pose depuis plusieurs décennies peut être repris à la lumière des analyses nouvelles qu'ont reçus le Tristan et la post-Vulgate, et des progrès réalisés dans la compréhension du fonctionnement des romans en prose. Ces éléments nous ont paru autoriser

qui est très étrange dans un cycle fondé en grande partie, d'après son éditrice (éd. cit. p. XXV), sur la rivalité de leur lignage et de celui de Gauvain, et alors que la Suite du Merlin, partie précédente du cycle, s'était déjà étendue sur les aventures de Tor. 
l'hypothèse d'une composition et d'une attribution complexes : le Tristan a repris à la Folie Lancelot le texte de péripéties dont il avait pourtant lui-même inventé l'essentiel. Le détail de cette opération demeure évidemment tout à fait hypothétique. Pourtant, reprendre les données relatives à ce problème engage également à reconsidérer la nature et la formation non pas seulement des épisodes concernés, mais la constitution même des cycles romanesques en prose, faits d'échanges et d'influences réciproques, et posant des questions d'identité et d'appartenance textuelles propres à ce type de récits. Ainsi les enfances de Perceval ne sont pas seulement représentatives des préoccupations littéraires et idéologiques du Tristan : elles illustrent également la mouvance particulière des textes en prose, difficiles à figer, impossibles à refermer, œuvres ouvertes 'en perpétuel devenir'. 